\title{
Angular multiplicity fluctuations in hadronic $Z$ decays and comparison to QCD models and analytical calculations
}

\section{L3 Collaboration}

M. Acciarri ${ }^{\text {aa }}$, O. Adriani ${ }^{\text {p }}$, M. Aguilar-Benitez ${ }^{\mathrm{z}}$, S. Ahlen ${ }^{\mathrm{k}}$, J. Alcaraz ${ }^{\mathrm{z}}$, G. Alemanni ${ }^{\mathrm{v}}$, J. Allaby ${ }^{\mathrm{q}}$, A. Aloisio ${ }^{\mathrm{ac}}$, M.G. Alviggi ${ }^{\text {ac }}$, G. Ambrosi ${ }^{\mathrm{s}}$, H. Anderhub aw , V.P. Andreev ${ }^{\text {al }}$, T. Angelescu ${ }^{\text {m }}$, F. Anselmo ', A. Arefiev ${ }^{\text {ab }}$, T. Azemoon ${ }^{\text {c }}$, T. Aziz ${ }^{\mathrm{j}}$, P. Bagnaia ${ }^{\text {ak }}$, L. Baksay ${ }^{\text {ar }}$, R.C. Ball ${ }^{\text {c }}$, S. Banerjee ${ }^{\mathrm{j}}$, Sw. Banerjee ${ }^{j}, K$. Banicz ${ }^{\text {at }}$, A. Barczyk ${ }^{\text {aw,au }}$, R. Barillère ${ }^{q}$, L. Barone ${ }^{\text {ak }}$, P. Bartalini ${ }^{\text {ah }}$, A. Baschirotto ${ }^{\text {aa }}$, M. Basile ${ }^{\text {i }}$, R. Battiston ${ }^{\text {ah }}$, A. Bay ${ }^{\mathrm{v}}$, F. Becattini ${ }^{\mathrm{p}}$, U. Becker ${ }^{\circ}$, F. Behner ${ }^{\text {aw }}$, J. Berdugo ${ }^{\mathrm{z}}$, P. Berges ${ }^{\circ}$, B. Bertucci ${ }^{\text {ah }}$, B.L. Betev ${ }^{\text {aw }}$, S. Bhattacharya ${ }^{j}$, M. Biasini ${ }^{q}$, A. Biland ${ }^{\text {aw }}$, G.M. Bilei a ${ }^{\text {ah }}$, J.J. Blaising ${ }^{d}$, S.C. Blyth ${ }^{\text {ai }}$, G.J. Bobbink ${ }^{\mathrm{b}}$, R. Bock ${ }^{\mathrm{a}}$, A. Böhm ${ }^{\mathrm{a}}$, L. Boldizsar ${ }^{\mathrm{n}}$, B. Borgia ${ }^{\text {ak }}$,

D. Bourilkov ${ }^{\text {aw }}$, M. Bourquin ${ }^{\text {s, D. Boutigny }}{ }^{\text {d }, ~ S . ~ B r a c c i n i ~}{ }^{\text {s }, ~ J . G . ~ B r a n s o n ~}{ }^{\text {an }}$, V. Brigljevic ${ }^{\text {aw }}$, I.C. Brock ${ }^{\text {ai }}$, A. Buffini ${ }^{p}$, A. Buijs ${ }^{\text {as }}$, J.D. Burger ${ }^{\circ}$, W.J. Burger ${ }^{\text {s }}$,

J. Busenitz ${ }^{\text {ar }}$, X.D. Cai ${ }^{\circ}$, M. Campanelli aw, M. Capell ${ }^{\circ}$, G. Cara Romeo ',

G. Carlino ${ }^{\text {ac }}$, A.M. Cartacci ${ }^{\text {p }}$, J. Casaus ${ }^{\mathrm{z}}$, G. Castellini ${ }^{\mathrm{p}}$, F. Cavallari ${ }^{\mathrm{ak}}$, N. Cavallo ${ }^{\text {ac }}$, C. Cecchi ${ }^{\text {s }}$, M. Cerrada ${ }^{\text {z }}$, F. Cesaroni ${ }^{\text {w }}$, M. Chamizo ${ }^{\text {z }}$, Y.H. Chang ay , U.K. Chaturvedi ', S.V. Chekanov ${ }^{\text {ae }}$, M. Chemarin ${ }^{\mathrm{y}}$, A. Chen ${ }^{\text {ay }}$, G. Chen ${ }^{\mathrm{g}}$, G.M. Chen ${ }^{\mathrm{g}}$, H.F. Chen ${ }^{\mathrm{t}}$, H.S. Chen ${ }^{\mathrm{g}}$, M. Chen ${ }^{\circ}$, G. Chiefari ${ }^{\mathrm{ac}}$, C.Y. Chien ${ }^{\mathrm{e}}$, L. Cifarelli ${ }^{\mathrm{am}}$, F. Cindolo ${ }^{\mathrm{i}}$, C. Civinini ${ }^{\mathrm{p}}$, I. Clare ${ }^{ }$, R. Clare ${ }^{\text {o, H.O. Cohn }}{ }^{\text {af }}$, G. Coignet ${ }^{\text {d }}$, A.P. Colijn ${ }^{\text {b }}$, N. Colino ${ }^{\text {z }}$, S. Costantini ${ }^{\text {h }}$, F. Cotorobai ${ }^{\mathrm{m}}$, B. de la $\mathrm{Cruz}^{\mathrm{z}}$, A. Csilling ${ }^{\mathrm{n}}$, T.S. Dai ${ }^{ }$, R. D'Alessandro ${ }^{\mathrm{p}}$, R. de Asmundis ${ }^{\text {ac }}$, A. Degré ${ }^{d}$, K. Deiters ${ }^{\text {au }}$, P. Denes ${ }^{\text {aj }}$, F. DeNotaristefani ${ }^{\text {ak }}$, D. DiBitonto ${ }^{\text {ar }}$, M. Diemoz ${ }^{\text {ak }}$, D. van Dierendonck ${ }^{\text {b }}$, F. Di Lodovico ${ }^{\text {aw }}$, C. Dionisi ${ }^{\text {ak }}$, M. Dittmar ${ }^{\text {aw }}$, A. Dominguez ${ }^{\text {an }}$, A. Doria ${ }^{\text {ac }}$, M.T. Dova ${ }^{\mathrm{r}, 1}$, E. Drago ${ }^{\text {ac }}$, D. Duchesneau ${ }^{d}$, P. Duinker ${ }^{b}$, I. Duran ${ }^{\text {ao }}$, S. Dutta ${ }^{j}$, S. Easo ${ }^{\text {ah }}$, Yu. Efremenko ${ }^{\text {af }}$, H. El Mamouni y , A. Engler ai, F.J. Eppling ${ }^{\circ}$, F.C. Erné b', J.P. Ernenwein ${ }^{y}$, P. Extermann ${ }^{\text {s }}$, M. Fabre ${ }^{\text {au }}$, R. Faccini ak, S. Falciano ${ }^{\text {ak }}$, A. Favara ${ }^{\text {p }}$, J. Fay ${ }^{y}$, O. Fedin ${ }^{\text {al }}$, M. Felcini ${ }^{\text {aw }}$, B. Fenyi ${ }^{\text {ar }}$, T. Ferguson ${ }^{\text {ai }}$, F. Ferroni ${ }^{\text {ak }}$, H. Fesefeldt ${ }^{\text {a }}$, E. Fiandrini ${ }^{\text {ah }}$, J.H. Field ${ }^{\text {s }}$, F. Filthaut ${ }^{\text {ai }}$, P.H. Fisher ${ }^{\circ}$, 
I. Fisk $^{\text {an }}$, G. Forconi ${ }^{\circ}$, L. Fredj ${ }^{\text {s }}$, K. Freudenreich ${ }^{\text {aw }}$, C. Furetta ${ }^{\text {aa }}$, Yu. Galaktionov ${ }^{\text {ab,o }}$, S.N. Ganguli j, P. Garcia-Abia ${ }^{\text {f }, ~ S . S . ~ G a u ~ ', ~ S . ~ G e n t i l e ~}{ }^{\text {ak }}$, J. Gerald ${ }^{\mathrm{e}}, \mathrm{N}$. Gheordanescu ${ }^{\mathrm{m}}$, S. Giagu ${ }^{\text {ak }}$, S. Goldfarb ${ }^{\mathrm{v}}$, J. Goldstein ${ }^{\mathrm{k}}$, Z.F. Gong ${ }^{\mathrm{t}}$, A. Gougas ${ }^{\mathrm{e}}$, G. Gratta ${ }^{\mathrm{ag}}$, M.W. Gruenewald ${ }^{\mathrm{h}}$, V.K. Gupta ${ }^{\text {aj }}$, A. Gurtu ${ }^{\text {j}}$, L.J. Gutay ${ }^{\text {at }}$, D. Haas ${ }^{\text {f }}$, B. Hartmann ${ }^{\text {a }}$, A. Hasan ${ }^{\text {ad }}$, D. Hatzifotiadou ${ }^{\text {i }}$, T. Hebbeker ${ }^{\text {h }}$, A. Hervé ${ }^{\text {q }}$, J. Hirschfelder ai, W.C. van Hoek ${ }^{\text {ae }}$, H. Hofer ${ }^{\text {aw }}$, H. Hoorani ${ }^{\text {ai }}$, S.R. Hou ${ }^{\text {ay }}$, G. Hu ${ }^{\text {e, }}$, V. Innocente ${ }^{q}$, K. Jenkes ${ }^{a}$, B.N. Jin ${ }^{g}$, L.W. Jones ${ }^{\text {c }}$, P. de Jong ${ }^{\text {q }}$, I. Josa-Mutuberria ${ }^{\mathrm{z}}$, A. Kasser ${ }^{\mathrm{v}}$, R.A. Khan ${ }^{\mathrm{r}}$, D. Kamrad ${ }^{\text {av }}$, Yu. Kamyshkov af, J.S. Kapustinsky ${ }^{x}$, Y. Karyotakis ${ }^{\text {d }}$, M. Kaur ${ }^{\text {r,2 }}$, M.N. Kienzle-Focacci ${ }^{\text {s, D. Kim }}{ }^{\text {ak }}$, D.H. Kim ${ }^{\text {aq }}$, J.K. Kim ${ }^{\text {aq }}$, S.C. Kim ${ }^{\text {aq }}$, W.W. Kinnison ${ }^{x}$, A. Kirkby ${ }^{\text {ag }}$, D. Kirkby ${ }^{\text {ag }}$, J. Kirkby ${ }^{\text {q }}$, D. Kiss ${ }^{\text {n }}$, W. Kittel ${ }^{\text {ae }}$, A. Klimentov ${ }^{\text {o,ab }}$, A.C. König ${ }^{\text {ae }}$, A. Kopp ${ }^{\text {av }}$, I. Korolko ${ }^{\text {ab }, ~ V . ~ K o u t s e n k o ~}{ }^{\text {o,ab }}$, R.W. Kraemer ${ }^{\text {ai }}$, W. Krenz ${ }^{\text {a }}$, A. Kunin ${ }^{\text {o,ab }}$, P. Lacentre ${ }^{\text {av, } 3,1}$,

P. Ladron de Guevara ${ }^{\mathrm{z}}$, G. Landi ${ }^{\mathrm{p}}$, C. Lapoint ${ }^{\circ}$, K. Lassila-Perini ${ }^{\mathrm{aw}}$, P. Laurikainen ", A. Lavorato ${ }^{a m}$, M. Lebeau ${ }^{\mathrm{q}}$, A. Lebedev ${ }^{\circ}$, P. Lebrun ${ }^{\mathrm{y}}$, P. Lecomte ${ }^{\text {aw }}$, P. Lecoq ${ }^{q}$, P. Le Coultre ${ }^{\text {aw }}$, H.J. Lee ${ }^{\text {h }}$, C. Leggett ${ }^{c}$, J.M. Le Goff ${ }^{q}$, R. Leiste ${ }^{\text {av }}$, E. Leonardi ak ${ }^{\text {a }}$ P. Levtchenko ${ }^{\text {al }}$, C. Li ${ }^{\mathrm{t}}$, C.H. Lin ${ }^{\text {ay }}$, W.T. Lin ${ }^{\text {ay }}$, F.L. Linde ${ }^{\mathrm{b}, \mathrm{q}}$, L. Lista ${ }^{\text {ac }}$, Z.A. Liu ${ }^{\mathrm{g}}$, W. Lohmann ${ }^{\text {av }}$, E. Longo ${ }^{\text {ak }}$, W. Lu ${ }^{\text {ag }}$, Y.S. Lu ${ }^{g}$, K. Lübelsmeyer ${ }^{\text {a }}$, C. Luci ${ }^{\text {ak }}$, D. Luckey ${ }^{\circ}$, L. Luminari ${ }^{\text {ak }}$, W. Lustermann ${ }^{\text {au }}$, W.G. Ma ${ }^{\mathrm{t}}$, M. Maity ${ }^{\mathrm{j}}$, G. Majumder ${ }^{\mathrm{j}}$, L. Malgeri ${ }^{\text {ak }}$, A. Malinin ${ }^{\text {ab }}$, C. Maña ${ }^{z}$, D. Mangeol ${ }^{\text {ae }}$, S. Mangla ${ }^{j}$, P. Marchesini ${ }^{\text {aw }}$, A. Marin ${ }^{\text {k }}$, J.P. Martin y, F. Marzano ak, G.G.G. Massaro b, D. McNally ${ }^{\text {q }}$, S. Mele ${ }^{\mathrm{q}}$, L. Merola $^{\text {ac }}$, M. Meschini ${ }^{\text {p }}$, W.J. Metzger ${ }^{\text {ae }}$, M. von der Mey ${ }^{\text {a }}$, Y. Mi ${ }^{\text {v }}$, D. Migani ${ }^{\mathrm{i}}$, A. Mihul ${ }^{\mathrm{m}}$, A.J.W. van Mil ${ }^{\text {ae }}, \mathrm{H}$. Milcent ${ }^{\mathrm{q}}$, G. Mirabelli ${ }^{\text {ak}}$, J. Mnich ${ }^{\mathrm{q}}$, P. Molnar ${ }^{\mathrm{h}}$, B. Monteleoni ${ }^{\mathrm{p}}$, R. Moore ${ }^{\mathrm{c}}, \mathrm{T}_{\text {. Moulik }}{ }^{\mathrm{j}}, \mathrm{R}_{\text {. Mount }}{ }^{\mathrm{ag}}$, F. Muheim ${ }^{\text {s }}$, A.J.M. Muijs ${ }^{\text {b }}$, S. Nahn ${ }^{\circ}$, M. Napolitano ${ }^{\text {ac }}$, F. Nessi-Tedaldi ${ }^{\text {aw }}$, H. Newman ${ }^{a g}$, T. Niessen ${ }^{a}$, A. Nippe ${ }^{v}$, A. Nisati ${ }^{\text {ak }}$, H. Nowak ${ }^{\text {av }}$, Y.D. Oh ${ }^{a q}$, H. Opitz ${ }^{\text {a }}$, G. Organtini ${ }^{\text {ak }}$, R. Ostonen ${ }^{\text {u }, ~ S . ~ P a l i t ~}{ }^{1}$, C. Palomares ${ }^{\text {z }}$, D. Pandoulas ${ }^{\text {a }}$, S. Paoletti ${ }^{\text {ak }}$, P. Paolucci ${ }^{\text {ac }}$, H.K. Park ${ }^{\text {ai }}$, I.H. Park ${ }^{\text {aq }}$, G. Pascale ${ }^{\text {ak }}$, G. Passaleva ${ }^{\text {q }}$, S. Patricelli ac, T. Paul ${ }^{1}$, M. Pauluzzi ah ${ }^{\text {a }}$, C. Paus ${ }^{\text {q }}$, F. Pauss ${ }^{\text {aw }}$, D. Peach ${ }^{\mathrm{q}}$, Y.J. Pei a , S. Pensotti aa, D. Perret-Gallix ${ }^{\text {d }}$, B. Petersen ${ }^{\text {ae }}$, S. Petrak ${ }^{\mathrm{h}}$, A. Pevsner ${ }^{\mathrm{e}}$, D. Piccolo ${ }^{\text {ac }}$, M. Pieri ${ }^{\mathrm{p}}$, P.A. Piroué ${ }^{\mathrm{aj}}$, E. Pistolesi ${ }^{\text {aa }}$, V. Plyaskin ${ }^{\text {ab }}$, M. Pohl ${ }^{\text {aw }}$, V. Pojidaev ab,p ${ }^{a}$, H. Postema ${ }^{\circ}$, N. Produit ${ }^{\mathrm{s}}$, D. Prokofiev al , J. Quartieri am, G. Rahal-Callot aw , N. Raja ${ }^{\text {j, P.G. Rancoita }}{ }^{\text {aa }}$, M. Rattaggi aa ${ }^{\text {a }}$, G. Raven ${ }^{\text {an }}$, P. Razis ${ }^{\text {ad }}$, K. Read af ${ }^{\text {, D. Ren }}{ }^{\text {aw }}$, M. Rescigno ${ }^{\text {ak }}$, S. Reucroft 1, T. van Rhee ${ }^{\text {as }}$, S. Riemann ${ }^{\text {av }}$, K. Riles ${ }^{c}$, O. Rind ${ }^{\mathrm{c}}$, A. Robohm ${ }^{\text {aw }}$, J. Rodin ${ }^{\circ}$, B.P. Roe ${ }^{\mathrm{c}}$, L. Romero ${ }^{\text {z, S. Rosier-Lees }}{ }^{\mathrm{d}}$, Ph. Rosselet ${ }^{\mathrm{v}}, \mathrm{W}$. van Rossum ${ }^{\text {as }}, \mathrm{S}$. Roth ${ }^{\text {a }}$, J.A. Rubio ${ }^{\mathrm{q}}$, D. Ruschmeier ${ }^{\text {h}}$, H. Rykaczewski ${ }^{\text {aw }}$, J. Salicio ${ }^{\text {q }}$, E. Sanchez ${ }^{\mathrm{z}}$, 
M.P. Sanders ${ }^{\text {ae }}$, M.E. Sarakinos ", S. Sarkar j, G. Sauvage ${ }^{\text {d }}$, C. Schäfer ${ }^{\text {a }}$, V. Schegelsky ${ }^{\text {al }}$, S. Schmidt-Kaerst ${ }^{\text {a }}$, D. Schmitz ${ }^{\text {a }}$, M. Schneegans ${ }^{\text {d }}$, N. Scholz ${ }^{\text {aw }}$, H. Schopper ax , D.J. Schotanus ${ }^{\text {ae }}$, J. Schwenke a , G. Schwering a , C. Sciacca ${ }^{\text {ac }}$, D. Sciarrino ${ }^{\text {s }}$, L. Servoli ${ }^{\text {ah }}$, S. Shevchenko ${ }^{a g}$, N. Shivarov ap, V. Shoutko ${ }^{a b}$, J. Shukla ${ }^{x}$, E. Shumilov ${ }^{\text {ab }}$, A. Shvorob ${ }^{\text {ag }}$, T. Siedenburg ${ }^{a}$, D. Son ${ }^{\text {aq }}$, V. Soulimov ${ }^{\text {ac }}$, B. Smith ${ }^{\circ}$, P. Spillantini ${ }^{\text {p }}$, M. Steuer ${ }^{\circ}$, D.P. Stickland ${ }^{\text {aj, }}$, H. Stone aj, B. Stoyanov ap , A. Straessner a, K. Sudhakar ${ }^{\text {i }}$, G. Sultanov ${ }^{\text {r }}$, L.Z. Sun ${ }^{\text {t }}$, G.F. Susinno ${ }^{\text {s, H. Suter }}{ }^{\text {aw }}$, J.D. Swain ${ }^{r}$, X.W. Tang ${ }^{g}$, L. Tauscher ${ }^{\text {f }}$, L. Taylor ${ }^{1}$, Samuel C.C. Ting ${ }^{o}$, S.M. Ting ${ }^{\circ}$, S.C. Tonwar ${ }^{j}$, J. Tóth ${ }^{n}$, C. Tully aj, H. Tuchscherer ${ }^{\text {ar }}$, K.L. Tung ${ }^{\text {g }}$, Y. Uchida ${ }^{\circ}$, J. Ulbricht ${ }^{\text {aw }}$, U. Uwer ${ }^{\mathrm{q}}$, E. Valente ${ }^{\mathrm{ak}}, \mathrm{G}$. Vesztergombi ${ }^{\mathrm{n}}$, I. Vetlitsky ${ }^{\mathrm{ab}}$, G. Viertel ${ }^{\mathrm{aw}}$, M. Vivargent ${ }^{\mathrm{d}}$, S. Vlachos ${ }^{\mathrm{f}}$, R. Völkert ${ }^{\text {av }}$, H. Vogel ${ }^{\text {ai }}$, H. Vogt ${ }^{\text {av }}$, I. Vorobiev ${ }^{\text {q,ab }}$, A.A. Vorobyov ${ }^{\text {al }}$, A. Vorvolakos ${ }^{\text {ad }}$, M. Wadhwa ${ }^{\text {f }}$, W. Wallraff ${ }^{\text {a }}$, J.C. Wang ${ }^{\circ}$, X.L. Wang ${ }^{\mathrm{t}}$, Z.M. Wang ${ }^{\mathrm{t}}$, A. Weber ${ }^{\mathrm{a}}$, S.X. Wu ${ }^{\mathrm{o}}$, S. Wynhoff ${ }^{\mathrm{a}}$, J. Xu ${ }^{\mathrm{k}}, \mathrm{Z} . Z$. Xu ${ }^{\mathrm{t}}$, B.Z. Yang ${ }^{\mathrm{t}}$, C.G. Yang ${ }^{\mathrm{g}}$, X.Y. Yao ${ }^{\mathrm{g}}$, J.B. Ye ${ }^{\mathrm{t}}$, S.C. Yeh ${ }^{\mathrm{az}}$, J.M. You ${ }^{\text {ai }}$, An. Zalite ${ }^{\text {al }}$, Yu. Zalite ${ }^{\text {al }}$, P. Zemp ${ }^{\text {aw }}$, Y. Zeng ${ }^{\text {a }}$, Z. Zhang ${ }^{\mathrm{g}}$, Z.P. Zhang ${ }^{\mathrm{t}}$, B. Zhou ${ }^{\text {k }}$, Y. Zhou ${ }^{\text {c }}$, G.Y. Zhu ${ }^{\text {g }}$, R.Y. Zhu ${ }^{\text {ag }}$, A. Zichichi i,q,r , F. Ziegler ${ }^{\text {av }}$

a I. Physikalisches Institut, RWTH, D-52056 Aachen, FRG ${ }^{4}$

III. Physikalisches Institut, RWTH, D-52056 Aachen, FRG ${ }^{4}$

${ }^{\mathrm{b}}$ National Institute for High Energy Physics, NIKHEF, and University of Amsterdam, NL-1009 DB Amsterdam, The Netherlands

' University of Michigan, Ann Arbor, MI 48109, USA

${ }^{\mathrm{d}}$ Laboratoire d'Annecy-le-Vieux de Physique des Particules, LAPP,IN2P3-CNRS, BP 110, F-74941 Annecy-le-Vieux CEDEX, France

e Johns Hopkins University, Baltimore, MD 21218, USA

${ }^{\mathrm{f}}$ Institute of Physics, University of Basel, $\mathrm{CH}-4056$ Basel, Switzerland

${ }^{\mathrm{g}}$ Institute of High Energy Physics, IHEP, 100039 Beijing, China ${ }^{5}$

${ }^{\mathrm{h}}$ Humboldt University, D-10099 Berlin, FRG ${ }^{4}$

${ }^{\mathrm{i}}$ University of Bologna and INFN-Sezione di Bologna, I-40126 Bologna, Italy

${ }^{\mathrm{j}}$ Tata Institute of Fundamental Research, Bombay 400005 , India

${ }^{\mathrm{k}}$ Boston University, Boston, MA 02215, USA

${ }^{1}$ Northeastern University, Boston, MA 02115, USA

${ }^{\mathrm{m}}$ Institute of Atomic Physics and University of Bucharest, R-76900 Bucharest, Romania

${ }^{n}$ Central Research Institute for Physics of the Hungarian Academy of Sciences, H-1525 Budapest 114, Hungary ${ }^{6}$

${ }^{\circ}$ Massachusetts Institute of Technology, Cambridge, MA 02139, USA

${ }^{\mathrm{p}}$ INFN Sezione di Firenze and University of Florence, I-50125 Florence, Italy

${ }^{\mathrm{q}}$ European Laboratory for Particle Physics, CERN, CH-1211 Geneva 23, Switzerland

${ }^{\mathrm{r}}$ World Laboratory, FBLJA Project, CH-1211 Geneva 23, Switzerland

${ }^{\mathrm{S}}$ University of Geneva, $\mathrm{CH}-1211$ Geneva 4, Switzerland

${ }^{\mathrm{t}}$ Chinese University of Science and Technology, USTC, Hefei, Anhui 230 029, China ${ }^{5}$

" SEFT, Research Institute for High Energy Physics, P.O. Box 9, SF-00014 Helsinki, Finland

"University of Lausanne, CH-1015 Lausanne, Switzerland

" INFN-Sezione di Lecce and Universitá Degli Studi di Lecce, I-73100 Lecce, Italy

${ }^{x}$ Los Alamos National Laboratory, Los Alamos, NM 87544, USA

${ }^{y}$ Institut de Physique Nucléaire de Lyon, IN2P3-CNRS, Université Claude Bernard, F-69622 Villeurbanne, France

${ }^{z}$ Centro de Investigaciones Energeticas, Medioambientales y Tecnologicas, CIFMAT, E-28040 Madrid, Spain ${ }^{7}$

aa INFN-Sezione di Milano, I-20133 Milan, Italy

ab Institute of Theoretical and Experimental Physics, ITEP, Moscow, Russia

ac INFN-Sezione di Napoli and University of Naples, I-80125 Naples, Italy

ad Department of Natural Sciences, University of Cyprus, Nicosia, Cyprus 


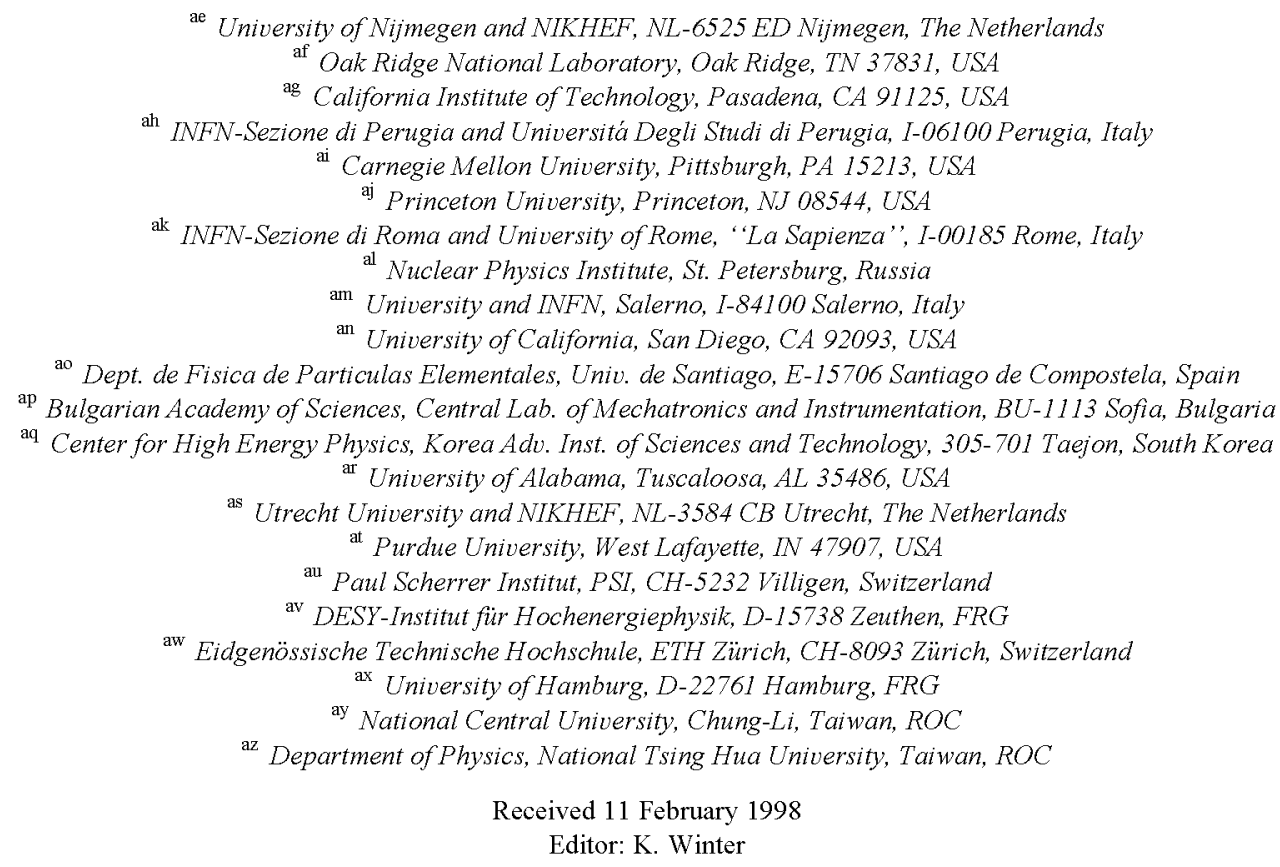

\section{Abstract}

Local multiplicity fluctuations in angular phase space intervals are studied using factorial moments measured in hadronic events at $\sqrt{s} \simeq 91.2 \mathrm{GeV}$, which were collected by the L3 detector at LEP. Parton shower Monte Carlo programs agree well with the data. On the other hand, first-order QCD calculations in the Double Leading Log Approximation and the Modified Leading Log Approximation are found to deviate significantly from the data. (C) 1998 Elsevier Science B.V. All rights reserved.

\section{Introduction}

The analytical perturbative approach (APA) to QCD jet physics combines perturbative QCD calcu-

\footnotetext{
${ }^{1}$ Also supported by CONICET and Universidad Nacional de La Plata, CC 67, 1900 La Plata, Argentina.

${ }^{2}$ Also supported by Panjab University, Chandigarh-160014, India.

${ }^{3}$ Supported by Deutscher Akademischer Austauschdienst.

${ }^{4}$ Supported by the German Bundesministerium für Bildung, Wissenschaft, Forschung und Technologie.

${ }^{5}$ Supported by the National Natural Science Foundation of China.

${ }^{6}$ Supported by the Hungarian OTKA fund under contract numbers T14459, T19181 and T24011.

${ }^{7}$ Supported also by the Comisión Interministerial de Ciencia y Technología.
}

lations [1] with the principle of Local Parton Hadron Duality (LPHD), which relates parton distributions to those of hadrons. LPHD [2] assumes that if the parton cascade is evolved down to a sufficiently low scale, hadronic distributions are proportional to partonic ones. All non-perturbative effects are thus reduced to a normalisation constant.

This approach has been quite successful in describing inclusive quantities such as the single-particle scaled momentum spectrum, $\xi=\ln \left(1 / x_{p}\right)$, and charged particle multiplicities in $\mathrm{e}^{+} \mathrm{e}^{-}$data at LEP energies. However, less inclusive quantities have met with less success [3].

In this paper we study local fluctuations of the charged particle multiplicity, which provides a new test of APA applied to many-particle inclusive densi- 
ties. Such fluctuations have been studied for many years in terms of a variety of phase space variables [4], but only recently has substantial progress been made in analytical QCD calculations of these observables [5-7].

We have recently investigated [8] local multiplicity fluctuations using bunching parameters [9], which showed directly that local fluctuations inside jets are multifractal, as is expected from QCD calculations [5-7]. In this paper we extend this study and present a quantitative comparison of first-order QCD calculations [5-7] with data from the L3 experiment at LEP using normalized factorial moments of orders $q=2, \ldots, 5$ in angular phase space intervals. The relative angle between particles has in the past proved to be sensitive to aspects of the QCD parton shower. For example, particle flow (the "string"' effect) [1012] and angular correlations such as the particle-particle correlation asymmetry (PPCA) [11,13] have demonstrated gluon interference in the parton shower.

An analysis similar to the present one [14] for $q=2,3$ found that calculations in the Double Leading Log Approximation (DLLA) [5] tended to underestimate the data if one used $\Lambda \simeq 0.1-0.2 \mathrm{GeV}$ for the QCD dimensional scale. However, reasonable agreement was found using an effective $\Lambda \simeq$ $0.04 \mathrm{GeV}$, which is very small compared to QCD estimates [15].

\section{Analytical calculations}

QCD calculations [6,7] for the normalized factorial moments (NFMs) [16], $F_{q}(\Theta)$, have the following scaling behavior

$$
\begin{aligned}
F_{q}(\Theta) & \equiv \frac{\langle n(n-1) \ldots(n-q+1)\rangle}{\langle n\rangle^{q}} \\
& \propto\left(\frac{\Theta_{0}}{\Theta}\right)^{\left(1-D_{q}\right)(q-1)},
\end{aligned}
$$

where $\Theta_{0}$ is the half opening angle of a cone around the jet-axis, $\Theta$ is the angular half-width window of rings around the jet-axis centered at $\Theta_{0}$ (see Fig. 1), and $n$ is the number of particles in these rings.

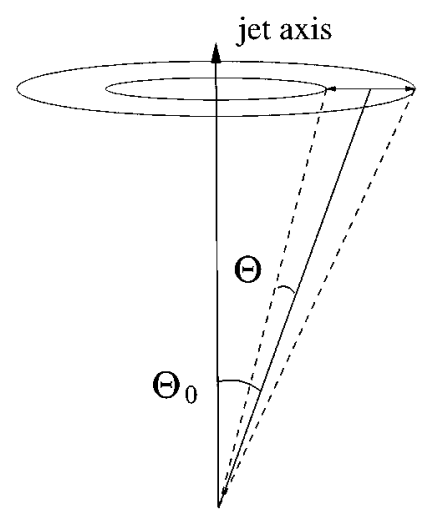

Fig. 1. A schematic representation of the measurements of the local fluctuations in the polar angle around jet axis $(D=1)$.

Brackets, \langle\rangle , around a quantity denote the average of that quantity over all events. Finally, $D_{q}$ is the so-called Rényi dimension. The analytical QCD expectations for $D_{q}$ are as follows [6,7]:

1. In the fixed-coupling regime, for moderately small angular bins,

$D_{q}=\gamma_{0}(Q) \frac{q+1}{q}$,

where $\gamma_{0}(Q)=\sqrt{2 C_{\mathrm{A}} \alpha_{\mathrm{s}}(Q) / \pi}$ is the anomalous QCD dimension calculated at $Q \simeq E \Theta_{0}, E$ $=\sqrt{s} / 2, s$ is the square of the center of mass energy, $\alpha_{\mathrm{s}}$ is the strong coupling constant, and $C_{\mathrm{A}}=3$ is the gluon color factor (equal to the number of colors).

2. In the running-coupling regime, for small bins, the Rényi dimension becomes a function of the size of the angular ring $\left(\alpha_{\mathrm{s}}(Q)\right.$ increases with decreasing $\Theta$ ).

It is useful to introduce a new scaling variable [7],

$z=\frac{\ln \left(\Theta_{0} / \Theta\right)}{\ln \left(E \Theta_{0} / \Lambda\right)}$.

The maximum possible phase space region $\left(\Theta=\Theta_{0}\right)$ corresponds to $z=0$.

There are three approximate expressions derived in DLLA which will be tested:

a) According to [6], the $D_{q}$ have the form

$$
D_{q} \simeq \gamma_{0}(Q) \frac{q+1}{q}\left(1+\frac{q^{2}+1}{4 q^{2}} z\right) \text {. }
$$


b) Another approximation has been suggested [7]:

$$
D_{q} \simeq 2 \gamma_{0}(Q) \frac{q+1}{q}\left(\frac{1-\sqrt{1-z}}{z}\right) \text {. }
$$

c) A result has also been obtained for the cumulant moments, which converge to factorial moments for high energies [5]:

$$
\begin{aligned}
& D_{q} \simeq 2 \gamma_{0}(Q) \frac{q-w(q, z)}{z(q-1)}, \\
& w(q, z)=q \sqrt{1-z}\left(1-\frac{\ln (1-z)}{2 q^{2}}\right) .
\end{aligned}
$$

Furthermore, an estimate for $D_{q}$ has been obtained in the Modified Leading Log Approximation (MLLA) [6]. In this case, Eq. (4) remains valid except that $\gamma_{0}(Q)$ is replaced by an effective $\gamma_{0}^{\text {eff }}(Q)$ depending on $q$ :

$$
\begin{aligned}
\gamma_{0}^{\text {eff }}(Q)= & \gamma_{0}(Q)+\gamma_{0}^{2}(Q) \frac{b}{4 C_{\mathrm{A}}}\left[-B \frac{q-1}{2(q+1)}\right. \\
& \left.+\frac{q-1}{2(q+1)\left(q^{2}+1\right)}+\frac{1}{4}\right]
\end{aligned}
$$

where

$b=\frac{11 C_{\mathrm{A}}}{3}-\frac{2 n_{\mathrm{f}}}{3}, \quad B=\frac{1}{b}\left[\frac{11 C_{\mathrm{A}}}{3}+\frac{2 n_{\mathrm{f}}}{3 C_{\mathrm{A}}^{2}}\right]$,

and $n_{\mathrm{f}}$ is the number of flavors.

For our comparison of the data with the theoretical calculations quoted above, we use the following parameters:

$n_{\mathrm{f}}=3, \quad \Lambda=0.16 \mathrm{GeV}$.

This value of $n_{\mathrm{f}}$ is chosen since even at high energies the production of heavy flavors will rarely happen in the jet and consequently its evolution is still dominated by the light flavors [17]. The value of $A$ chosen is that found in tuning the JETSET 7.4 matrix element program [18] on L3 data [19] and in our recent determination of $\alpha_{\mathrm{s}}\left(m_{\mathrm{Z}}\right)$ [20].

For the angle $\Theta_{0}$, we consider two possibilities: $\Theta_{0}=25^{\circ}$ and $35^{\circ}$. The first value, suggested by authors of two of the calculations [21], is the same as used in the DELPHI analysis [14]. The larger value of $\Theta_{0}$ allows a larger range of $\Theta$ to be studied.

The effective coupling constant is evaluated at $Q=E \Theta_{0}$. For $\Theta_{0}=25^{\circ}$, one obtains $\alpha_{\mathrm{s}}\left(E \Theta_{0}\right)=$
0.144 according to the first-order QCD expression for $\alpha_{\mathrm{s}}(Q)$. This value leads to $\gamma_{0}\left(E \Theta_{0}\right)=0.525$. For $\Theta_{0}=35^{\circ}, \alpha_{\mathrm{s}}\left(E \Theta_{0}\right)=0.135$ and $\gamma_{0}\left(E \Theta_{0}\right)=0.508$.

\section{Experimental procedure}

The analysis is based on data, corresponding to an integrated luminosity of $52 \mathrm{pb}^{-1}$, collected by the L3 detector [22] at a center of mass energy of $\sqrt{\mathrm{s}}=$ 91.2 GeV during the 1994 LEP running period. Hadronic events are selected using information from the Central Tracking Detector (TEC) and the Silicon Microvertex Detector (SMD).

To obtain a sample with well-measured charged tracks, a selection is performed using tracks which have passed certain quality cuts. To ensure that the event lies within the full acceptance of the TEC and $\mathrm{SMD}$, the direction of the thrust axis, as determined from the charged tracks, must satisfy $\left|\cos \theta_{\text {thr }}\right|<0.7$. Events are then selected using the following criteria:

$$
\begin{aligned}
& \frac{\sum_{i}\left|p_{i}\right|}{\sqrt{s}}>0.15, \quad \frac{\left|\sum_{i} p_{\| i}\right|}{\sum_{i}\left|p_{i}\right|}<0.75, \\
& \frac{\left|\sum_{i} p_{\perp i}\right|}{\sum_{i}\left|p_{i}\right|}<0.75, \quad N_{\mathrm{ch}}>4,
\end{aligned}
$$

where $p_{i}$ is the momentum of particle $i$ and the sum runs over all tracks of an event, and where $N_{\mathrm{ch}}$ is the number of charged tracks. The resulting sample contains about 1.0 million events.

In this paper we study fluctuations in small angular bins. For the grouping of tracks into these bins, the resolution of the angle between pairs of tracks is of crucial importance. For this reason we impose additional stringent quality cuts on track reconstruction, which results in rejection of $39 \%$ of the tracks. With this selection we achieve very good agreement between data and simulation for the distributions of the difference in angle between pairs of tracks for both the azimuthal angle about, and the polar angle with respect to, the beam [8]. 
An NFM calculated from the data is corrected for detector effects by a correction factor determined from two Monte Carlo samples. Events generated with the JETSET 7.4 parton shower (PS) program [18] including initial-state photon radiation are passed through a full detector simulation [23] including time-dependent variations of the detector response based on continuous detector monitoring and calibration. It has been reconstructed with the same program as the data and passed through the same selection procedure. The resulting sample is referred to as detector level MC. Another sample, called generator level $\mathrm{MC}$, is generated directly from JETSET. It contains all charged final-state particles with a lifetime $c \tau>1 \mathrm{~cm}$ and is generated without initial-state photon radiation, Dalitz pairs or Bose-Einstein correlations, since these effects are not included in the analytical QCD calculations.

From these two samples a correction factor is found: $C_{q}=F_{q}^{\text {gen }} / F_{q}^{\text {det }}$, where $F_{q}^{\text {gen }}$ and $F_{q}^{\text {det }}$ are the values of the NFM of order $q$ calculated from the generator level and detector level, respectively. The corrected NFM is then given by $F_{q}=C_{q} F_{q}^{\text {raw }}$, where $F_{q}^{\text {raw }}$ is the NFM calculated directly from the data. The correction is of the order of $3 \%$ for $F_{2}$, increasing to approximately $5 \%$ for $F_{5}$.

The resolution of the L3 detector for a number of relevant variables has been estimated [24]. The resolution of polar angle defined with respect to the thrust axis is found to be approximately 0.01 radians. For higher orders NFMs, the minimum angle $\Theta$ used in this study is chosen according to the many-particle resolutions studied in [24].

The errors on the results include both statistical and systematic errors on the raw quantities and on the correction factors. The systematic errors on the raw quantities, found from variation of track quality cuts and event selection cuts were found to be negligible. The systematic error on the correction factors is taken as half of the difference between the correction factors determined using JETSET and those using HERWIG 5.9 [25].

\section{Results}

The sphericity axis is used to define the jet axis. To increase statistics, we evaluated the NFMs in each sphericity hemisphere of an event and averaged
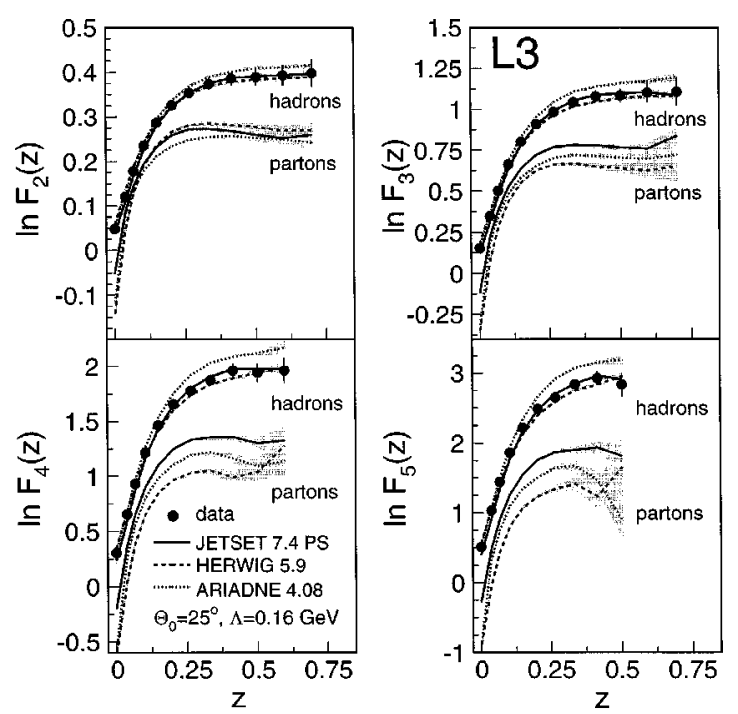

Fig. 2. NFMs, $F_{q}(z),(q=2, \ldots, 5)$ as a function of the scaling variable $z$, for $\Theta_{0}=25^{\circ}$ and $A=0.16 \mathrm{GeV}$, compared to Monte Carlo model predictions on the partonic and hadronic levels. The shaded areas in this figure, and in Fig. 3Fig. 4, represent the statistical errors on the model predictions.

the results, thus assuming that the local fluctuations in each hemisphere are independent. Fig. 2 shows the experimental results on the behavior of the NFMs as a function of the scaling variable $z$ for $\Theta_{0}=25^{\circ}$ and $\Lambda=0.16 \mathrm{GeV}$. The data were corrected using the method discussed above. The error bars include statistical and systematical errors.

\subsection{Comparison with Monte Carlo models}

The data in Fig. 2 are compared with the predictions of the JETSET, HERWIG, and ARIADNE [26] parton shower models at both the hadronic and partonic levels. All three models have been tuned to reproduce global event-shape and single-particle inclusive distributions ${ }^{8}$ [27,28]. The hadronic-level predictions of the models give a good description of the fluctuations. The effect of heavy flavors ( $c$ and $b$ quarks) has been estimated by rejecting these flavors in JETSET. The effect was found to be negligible.

\footnotetext{
${ }^{8}$ The Bose-Einstein modelling of JETSET is used in ARIADNE; HERWIG contains no Bose-Einstein model; and JETSET was used with its Bose-Einstein modelling turned off.
} 
The data and the hadronic level of the models saturate later than does the partonic level.

It is expected [7] that hadronization effects would largely cancel in the ratio $F_{q}(z) / F_{q}(0)$. In addition, this ratio eliminates a theoretical ambiguity in the normalization of the NFMs, i.e., in $F_{q}(0)$. In terms of $F_{q}(z) / F_{q}(0)$, the power law of Eq. (1) can be rewritten as

$\ln \frac{F_{q}(z)}{F_{q}(0)}=z\left(1-D_{q}\right)(q-1) \ln \frac{E \Theta_{0}}{\Lambda}$.

The behavior of $\ln \left(F_{q}(z) / F_{q}(0)\right)$ as a function of $z$ is shown in Figs. 3 and 4 for the partonic and hadronic levels, respectively. The partonic level predictions of the models are indeed much closer to the data, and the differences between partonic and hadronic levels are decreased, particularly for the higher-order moments. Here too the hadronic level of the models provides a satisfactory description of the data. The degree of similarity between partonic and hadronic level MC predictions can be interpreted as a measure of the degree of validity of LPHD. We note that there is a greater difference between the partonic and hadronic levels of HERWIG than of JETSET with ARIADNE lying in between. We also note that the average number of partons is about 8.6,
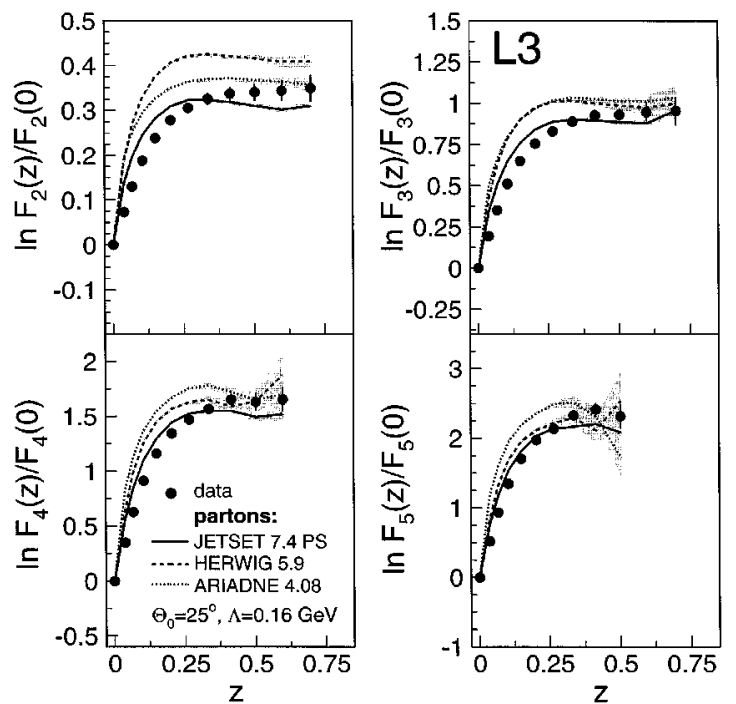

Fig. 3. $F_{q}(z) / F_{q}(0)(q=2, \ldots, 5)$ as a function of the scaling variable $z$, for $\Theta_{0}=25^{\circ}$ and $\Lambda=0.16 \mathrm{GeV}$, compared to Monte Carlo model predictions on the partonic level.
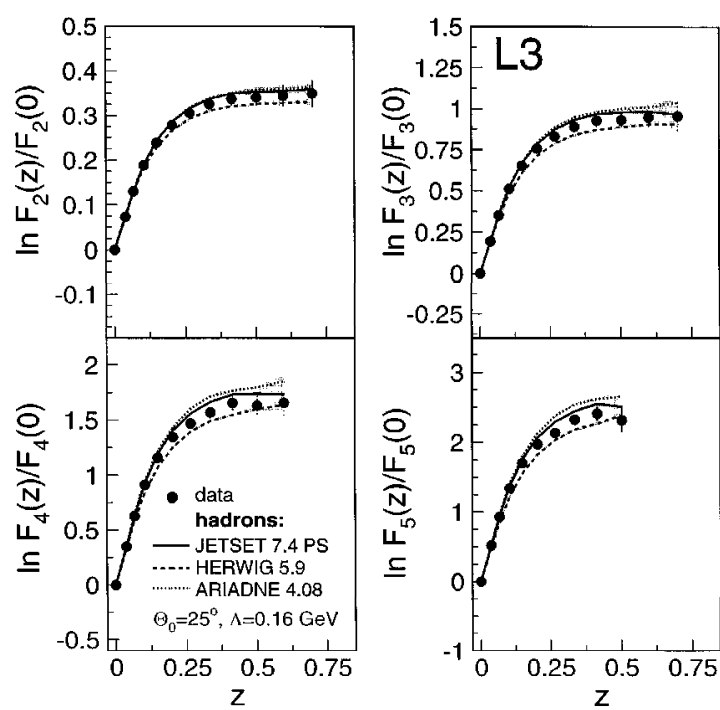

Fig. 4. $F_{q}(z) / F_{q}(0)(q=2, \ldots, 5)$ as a function of the scaling variable $z$, for $\Theta_{0}=25^{\circ}$ and $A=0.16 \mathrm{GeV}$, compared to Monte Carlo model predictions on the hadronic level.

10.0, and 10.2 for HERWIG, JETSET, and ARIADNE, respectively.

\subsection{Comparison with analytical calculations}

The comparison of the analytical QCD calculations (Eqs. (2), (4)-(7)) with the corrected data is shown in Fig. 5 for $A=0.16 \mathrm{GeV}$ and $\Theta_{0}=25^{\circ}$. For the second order moment, running $\alpha_{\mathrm{s}}$ calculations lead to the saturation effects observed in the data, but significantly underestimate the observed signal. Predictions for the higher moments are too low for low values of $z$, but tend to overestimate the data at larger $z$. The fixed coupling regime (thin solid lines) approximates the running coupling regime for small $z$, but does not exhibit the saturation effect seen in the data. The DLLA approximations (Eqs. (4)-(6)) differ significantly at large $z$, with the calculations from cumulants (Eq. (6)) showing the strongest saturation effect. The MLLA predictions are rather similar to the DLLA results of Eq. (4).

We have also compared (not shown) the data and the QCD predictions for $\Theta_{0}=35^{\circ}$. Both the data and the predictions rise more rapidly than for $\Theta_{0}=25^{\circ}$. This indicates that fluctuations are larger for phase space regions containing a larger contribution from hard gluon radiation. However, the disagreement 

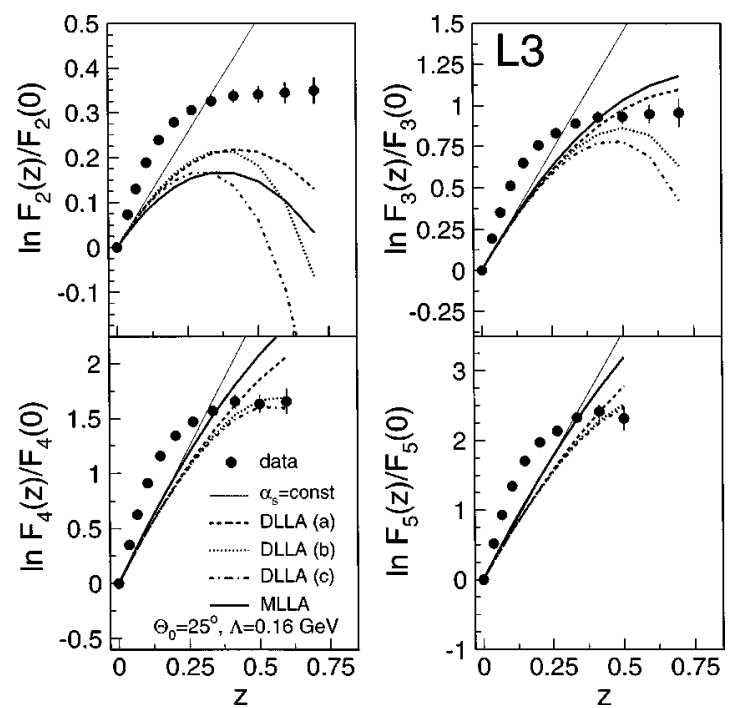

Fig. 5. $F_{q}(z) / F_{q}(0)(q=2, \ldots, 5)$ as a function of the scaling variable $z$, for $\Theta_{0}=25^{\circ}$ and $A=0.16 \mathrm{GeV}$, compared to the analytical QCD calculations for: $\alpha_{\mathrm{s}}=$ const (Eq. 2); DLLA (a) (Eq. 4); DLLA (b) (Eq. 5); DLLA (c) (Eq. 6); and MLLA (Eq. 7 ).

between data and predictions is similar to that for $\Theta_{0}=25^{\circ}$.

In a study using the parameterization of [5] (Eq. (6)), DELPHI has found better agreement with their
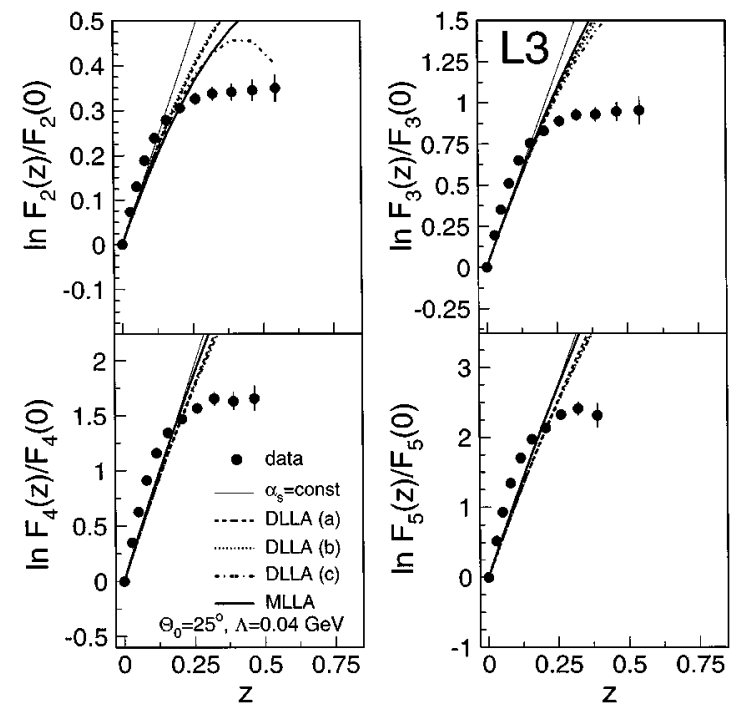

Fig. 6. $F_{q}(z) / F_{q}(0)(q=2, \ldots, 5)$ as a function of the scaling variable $z$, for $\Theta_{0}=25^{\circ}$ and $A=0.04 \mathrm{GeV}$, compared to the analytical QCD calculations for: $\alpha_{\mathrm{s}}=$ const (Eq. 2); DLLA (a) (Eq. 4); DLLA (b) (Eq. 5); DLLA (c) (Eq. 6); and MLLA (Eq. 7). data by decreasing the value of $\Lambda$ to $0.04 \mathrm{GeV}$ [14] A smaller effective value makes the coupling constant smaller, which expands the range of validity of the perturbative calculations (for $\Lambda=0.04 \mathrm{GeV}$, $\left.\alpha_{\mathrm{s}}\left(E \Theta_{0}\right)=0.112, \gamma_{0}\left(E \Theta_{0}\right)=0.46\right)$. Fig. 6 shows the case of $\Lambda=0.04 \mathrm{GeV}$ for our data. While the agreement for small $z$ is indeed better, it becomes worse for $z>0.3$, where contributions from higherorder perturbative QCD and hadronization are expected to be larger. We have varied $\Lambda$ in the range of $0.04-0.25 \mathrm{GeV}$ and found that there is no value of $\Lambda$ in this range which produces agreement for all orders of NFMs. Increasing the number of active flavors, $n_{\mathrm{f}}$, to 4 or 5 leads to worse agreement.

\subsection{Discussion}

The first-order calculations of the DLLA and MLLA of perturbative QCD are shown to be in disagreement with the local fluctuations observed in hadronic $Z$ decay. This occurs both for standard values of $A(A=0.16 \mathrm{GeV})$ and for small values ( $A=0.04 \mathrm{GeV})$. In the latter case, a reasonable estimate for $z<0.3$ can be obtained, consistent with the DELPHI conclusion [14]. However, in this case, the theoretical NFMs strongly overestimate the data for relatively large $z$ (small $\Theta$ ), where contributions from higher-order perturbative QCD are larger.

On the other hand, the MC models all agree well with the data.

Likely reasons for the failure of the calculations are their asymptotic character, which corresponds to an infinite number of partons in an event, and their lack of energy-momentum conservation, features which are taken into account in the MC models. A similar conclusion was reached from a comparison of factorial and cumulant moments in quark and gluon jets [29]. Further, a recent theoretical study [30] of energy conservation in triple-parton vertices shows that the energy conservation constraint is indeed sizeable and leads to a stronger saturation effect. Note that the MLLA predictions used here are not from a full MLLA calculation. This MLLA calculation only modifies $\gamma_{0}$, while retaining the DLLA parameterization of the $z$-dependence of the NFMs, which is only asymptotically correct.

Another contribution to the failure of the predictions can lie with the local parton-hadron duality 
hypothesis, which is used to justify comparison of the analytical QCD calculations with hadronic data. In JETSET the difference between parton- and particle-level predictions are large for $F_{q}(z)$ but small for $F_{q}(z) / F_{q}(0)$. However, for HERWIG and ARIADNE this is not the case. At the shower cut-off scales of these models, the hadronization effects are thus still important and depend on $z$. Thus LPHD does not apply at these cut-off scales. It is conceivable that lowering the cut-off in these models below the current value of about $1 \mathrm{GeV}$ would result in smaller hadronization effects and better agreement. However, we consider it unlikely that such a model could successfully describe other aspects of the data, such as production rates for baryons and high-mass meson resonances.

\section{Conclusions}

Monte Carlo models incorporating a coherent parton shower agree well with the data. On the other hand, first-order calculations in the DLLA and MLLA of perturbative QCD disagree with the local fluctuations observed in hadronic $Z$ decay. The asymptotic nature of the calculations and their inadequate treatment of energy-momentum conservation appear to be the most likely reasons for the failure of the calculations. The Monte Carlo results at parton level indicate that the influence of hadronization is not in agreement with the LPHD assumption.

\section{Acknowledgements}

We wish to express our gratitude to the CERN accelerator divisions for the excellent performance of the LEP machine. We acknowledge the effort of all the engineers and technicians who have participated in the construction and maintenance of this experiment. We thank B. Buschbeck, M. Chmeissani, I. Dremin, V.I. Kuvshinov, P. Lipa, F. Mandl, J.-L. Meunier, P. Nason, W. Ochs, R. Peschanski, T. Sjöstrand and B. Webber for helpful discussions.

\section{References}

[1] Yu. Dokshitzer, V.A. Khoze, S.I. Troyan, A.H. Mueller, Rev. Mod. Phys. 60 (1988) 373; Yu.L. Dokshitzer, V.A.
Khoze, A.H. Mueller, S.I. Troyan, Basics of Perturbative QCD, Editions Frontières, 1991.

[2] Ya.I. Azimov, Yu.L. Dokshitzer, V.A. Khoze, S.I. Troyan, Z. Phys. C 27 (1985) 65.

[3] V.A. Khoze, W. Ochs, Int. J. Mod. Phys. A 12 (1997) 2949

[4] P. Bożek, M. Płoszajczak, R. Botet, Phys. Rep. 252 (1995) 101; E.A. De Wolf, I.M. Dremin, W. Kittel, Phys. Rep. 270 (1996) 1

[5] W. Ochs, J. Wosiek, Phys. Lett. B 289 (1992) 159; B 304 (1993) 144; Z. Phys. C 68 (1995) 269.

[6] Yu. Dokshitzer, I.M. Dremin, Nucl. Phys. B 402 (1993) 139.

[7] Ph. Brax, J.-L. Meunier, R. Peschanski, Z. Phys. C 62 (1994) 649.

[8] L3 Collab., Local multiplicity fluctuations in hadronic $Z$ decay, CERN-PPE/97-137, submitted to Phys. Lett. B.

[9] S.V. Chekanov, V.I. Kuvshinov, Acta Phys. Polonica B 25 (1994) 1189; S.V. Chekanov, W. Kittel, V.I. Kuvshinov, Acta Phys. Polonica B 27 (1996) 1739; Z. Phys. C 73 (1997) 517.

[10] JADE Collab., W. Bartel et al., Phys. Lett. B 101 (1981) 129; Z. Phys. C 21 (1983) 37; TPC/2 $\gamma$ Collab., Z. Phys. C 28 (1985) 31; TASSO Collab., M. Althoff et al., Z. Phys. C 29 (1985) 29; MARK II Collab., P.D. Sheldon et al., Phys. Rev. Lett. 57 (1986) 1398; DELPHI Collab., P. Aarnio et al., Phys. Lett. B 240 (1990) 271; OPAL Collab., M.Z. Akrawy et al., Phys. Lett. B 261 (1991) 334; L3 Collab., M. Acciarri et al., Phys. Lett. B 345 (1995) 74.

[11] ALEPH Collab., Studies of Quantum Chromodynamics with the ALEPH Detector, CERN-PPE /96-186, submitted to Phys. Rep.

[12] Ya.I. Azimov, Yu.L. Dokshitzer, V.A. Khoze, S.I. Troyen, Phys. Lett. B 165 (1985) 147.

[13] M. Chmeissani, I.F.A.E., Universitat Autonoma de Barcelona, Annual Report, 1992, p. 49; Aly Aamer Syed, Particle Correlations in Hadronic Decays of the ZBoson, Ph.D. Thesis, Univ. of Nijmegen, The Netherlands, 1994, ISBN 909007038-9; L3 Collab., M. Acciarri et al., Phys. Lett. B 353 (1995) 145

[14] B. Buschbeck, F. Mandl, DELPHI Collaboration, ICHEP'96 contributed paper pa01-028.

[15] Particle Data Group, R.M. Barnett et al., Physics Phys. Rev. D 54 (1996) 1.

[16] A. Białas, R. Peschanski, Nucl. Phys. B 273 (1986) 703; B 308 (1988) 857.

[17] S. Lupia, W. Ochs, Phys. Lett. B 365 (1996) 339; see also: hep-ph/9704319.

[18] T. Sjöstrand, Comp. Phys. Comm. 82 (1994) 74.

[19] S. Banerjee, D. Duchesneau, S. Sarkar, L3 Note 1818 (1995); J. Casaus, L3 Note 1946 (1996); L3 Collab., B. Adeva et al., Z. Phys. C 55 (1992) 39.

[20] A. Buytenhuijs, QCD Gluon Radiation Studies Using the L3 Detector, Ph.D. Thesis, Univ. of Nijmegen, The Netherlands, 1996, ISBN 90-9009974-3.

[21] J.-L. Meunier, W. Ochs, private communications.

[22] L3 Collab., B. Adeva et al., Nucl. Inst. Meth. A 289 (1990) 35; J.A. Bakken et al., Nucl. Inst. Meth. A 275 (1989) 81; O. Adriani et al., Nucl. Inst. Meth. A 302 (1991) 53; B. Adeva 
et al., Nucl. Inst. Meth. A 323 (1992) 109; K. Deiters et al., Nucl. Inst. Meth. A 323 (1992) 162; B. Acciari et al., Nucl. Inst. Meth. A 351 (1994) 300.

[23] The L3detector simulation is based on GEANT3, see R. Brun et al., CERN report CERN DD/EE/84-1 (Revised), 1987, and uses GHEISHato simulate hadronic interactions, see $\mathrm{H}$. Fesefeldt, RWTH Aachen report PITHA 85/02, 1985.

[24] S.V. Chekanov, Local Multiplicity Fluctuations and Intermittent Structure Inside Jets, Ph.D. Thesis, Univ. of Nijmegen, The Netherlands, 1997, ISBN 90-9010518-2.
[25] G. Marchesini, B.R. Webber, Nucl. Phys. B 310 (1988) 461; G. Marchesini et al., Comp. Phys. Comm. 67 (1992) 465.

[26] L. Lönnblad, Comp. Phys. Comm. 71 (1992) 15.

[27] L3 Collab., B. Adeva et al., Z. Phys. C 55 (1992) 39.

[28] S. Banerjee, S. Banerjee, L3 Note 1978, 1996; I.G. Knowles, T. Sjöstrand (conveners), QCD Event Generators, Physics at LEP2, CERN-96-01, vol. 2, 1996, p. 103.

[29] OPAL Collaboration, K. Ackerstaff et al., CERN-PPE/97105 (Submitted to Z. Phys. C).

[30] J.-L. Meunier, R. Peschanski, Z. Phys. C 72 (1996) 647. 